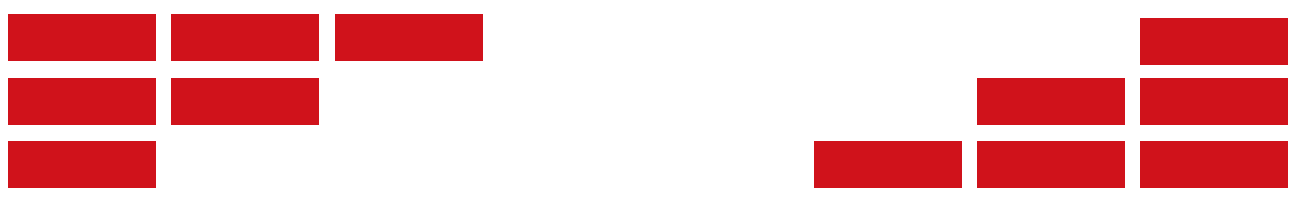

\title{
Inseguridad y juegos evolutivos
}

\section{Public insecurity and evolutionary games}

Raúl Vladimir Gómez Díaz

Universidad de Guadalajara, México rgomez1538@outlook.es

Carlos Obed Figueroa Ortiz

Universidad de Guadalajara, México figueroac@cucea.udg.mx 


\section{Resumen}

Esta investigación utiliza la teoría de los juegos evolutivos para determinar la importancia de la seguridad pública y las intervenciones gubernamentales para alcanzar un máximo de bienestar social cuando prevalece la inseguridad. El método de estudio consiste en modelar un juego evolutivo propietario-oportunista con altos niveles de delincuencia y poca acción de las víctimas, e ir alternando con las intervenciones del gobierno. Se concluye que el único mecanismo a través del cual el gobierno puede hacer que la población alcance un máximo de bienestar social es mejorando la seguridad pública, independientemente de si existen mayores penalidades para los delincuentes.

Palabras Clave: juegos evolutivos, inseguridad, economía del crimen, dinámica del replicador.

\section{Abstract}

This research uses evolutionary game theory to determine the importance of public safety and government interventions to maximize social welfare when insecurity prevails. The study method consists of modeling an owner-opportunistic evolutionary game with high levels of crime and little action by the victims, alternating with government interventions. Conclusions show that the only mechanism through which the government can make the population achieve a maximum of social welfare is by improving public security, regardless of whether there are greater penalties for criminals.

Keywords: evolutionary games, insecurity, crime economics, replicator dynamics.

Clasificación JEL: C72, C73, K42
Fecha de recepción: 12 de noviembre de 2021.

Fecha de aceptación: 26 de enero de 2022.

Fecha de publicación: 31 de enero de 2022. 


\section{Introducción}

Las investigaciones sobre el crimen son bastante extensas y diferenciadas entre sí, concluyentes en algunas ideas, pero sin lograr una unificación. Si se aborda el crimen como un fenómeno sociológico se encontrará que la estructura bajo la cual se desarrolla el individuo es clave para que este tome la decisión de delinquir o no; mientras que en el enfoque racional (Becker, 1968) se propone que todos podemos cometer actividades delictivas; sin embargo, no actuamos de esa manera debido a que nuestro análisis costo-beneficio no es favorable. También existen estudios mediante la teoría de juegos, en los que se plantea que toda actividad ilegal es una situación (juego) en la que dos personas (jugadores) se enfrentan y toman acción (estrategia) en un momento determinado. Si bien se utilizará este último enfoque, existe un campo más específico y completo: los juegos evolutivos, en los cuales los agentes no solo se enfrentan de manera estática, sino que cumplen con un proceso de aprendizaje a lo largo del tiempo, crean una dinámica entre la población.

Para el enfoque de la economía del crimen se tiene como pionero a Becker (1968), quien plantea que todos los posibles delincuentes enfrentan un problema de análisis costo-beneficio en el que consideran variables como vigilancia, penalidades y el valor del posible robo, mientras que la sociedad asume el costo del mantenimiento de cárceles, el pago de impuestos para mantener la vigilancia y el bien perdido. Encuentra dos curvas, una de ingreso marginal y otra de costo marginal, y concluye con un modelo en el cual ambos jugadores interactúan por medio del número de delitos.

Otro enfoque de la economía del crimen es el mercado de ofensas (Ehrlich, 1996), en el que los criminales ofertan delitos y la sociedad los demanda. La curva de oferta se plantea a partir de componentes como el costo del crimen, el salario legítimo que se pierde y el producto de la probabilidad de aprehensión por la pena de ser condenado, dando como resultado que a mayor cantidad de crímenes corresponde un mayor beneficio para los delincuentes; mientras que la curva de demanda es una relación negativa entre tolerancia y número de delitos, en la cual a mayor número de crímenes la sociedad tendrá menos tolerancia y, como consecuencia, habría un gasto mayor en autoprotección.

Hasta ahora se ha podido observar que existe una dinámica entre propietarios y delincuentes a través del número de crímenes, niveles de protección y vigilancia de la policía tal, que se evidencia un comportamiento estratégico entre ambos; esto nos lleva a la teoría de juegos evolutiva, planteando modelos bajo una dinámica del replicador (Cohen y Machalek, 1988; Cressman et al., 1998; Machalek 
y Cohen, 1991; Mealey, 1997; Savage y Vila, 2003; Vila, 1994, 1995, 1997; Vila y Cohen, 1993; Weissing y Ostrom, 1991). Una consideración que se debe hacer sobre el criminal es que este, al tomar una decisión, tiene más de una estrategia; es decir, que las condiciones hacen que opte por cometer un crimen, por lo cual tanto el criminal como la posible víctima están observando sus posibles estrategias, existiendo así una interacción. Por eso, el crimen tendrá uno o varios equilibrios de Nash (1950), los cuales a su vez podrían ser considerados estrategias evolutivamente estables si es que cumplen con la condición de estabilidad.

El juego está especificado de la siguiente manera; existe un gobierno que busca reducir la actividad delictiva por medio de dos mecanismos: aumento de las penas y nivel de patrullaje (Becker, 1968). Ambos implican un gasto para la sociedad, razón por la cual se busca que incurran en el menor costo y la mayor efectividad. Los jugadores son de dos poblaciones; por un lado, criminales definidos como oportunistas, quienes tendrán optan por robar o no robar, según sea la oportunidad que se presente; por otro, están los propietarios, quienes deciden entre protegerse y no hacerlo, lo cual depende de su percepción y aprendizaje, dados los costos de protección y la probabilidad de sufrir un robo. Lo anterior se desarrolla en un juego evolutivo conforme a un emparejamiento aleatorio entre jugadores de diferentes poblaciones con un proceso de constante aprendizaje, que concluye en la imitación de la estrategia que consideran más exitosa.

El objetivo principal que persigue esta investigación es estudiar qué implicaciones tiene un estado de inseguridad sobre la evolución de las estrategias entre propietarios y oportunistas, a través de la dinámica del replicador y las modificaciones que esto tiene sobre los niveles de bienestar. Como objetivo secundario se implementa una intervención gubernamental, con la finalidad de transitar hacia un estado de paz, en el que no sea necesario pagar por seguridad privada y los oportunistas no tengan incentivos de robar. Esta intervención consiste en desincentivar al oportunista a través de un aumento en las penas y en los niveles de seguridad pública.

La motivación de esta investigación es profundizar en el comportamiento de las posibles víctimas y delincuentes, a través de los incentivos cuando existe efectividad privada total y efectividad pública parcial. La finalidad es evaluar el impacto de la policía pública aislado del efecto de seguridad privada, de tal manera que se puede determinar qué tan relevante es el papel de la policía cuando el juego se encuentra en un estado de inseguridad y si esta desempeña un papel fundamental para alcanzar un estado de paz. 


\section{Modelo}

Este apartado se divide en dos: la primera parte consiste en explicar el modelo simple propietario-oportunista (Cressman et al., 1998) y obtener sus equilibrios de Nash, y después introducir a la policía pública y volver a obtener los nuevos equilibrios de Nash, así como los beneficios esperados, los cuales serán definidos a lo largo del trabajo como niveles de bienestar. En la segunda parte, con el modelo completo, se obtienen las ecuaciones del replicador y se definen los criterios para determinar los estados estacionarios del sistema.

Se plantea un primer juego poblacional entre propietarios y oportunistas, en el que los propietarios tienen dos estrategias: pagar seguridad privada para evitar un robo (activo) o no hacerlo y correr el riesgo (pasivo); mientras que los oportunistas deciden entre robar y no robar. Es importante destacar que en este juego no existe ningún otro medio para evitar un robo que la seguridad privada.

En un segundo modelo se introduce a la seguridad pública como mecanismo de disuasión del robo para propietarios que deciden no pagar por seguridad privada. En este nuevo juego la seguridad pública será pagada por medio de impuestos de ambas poblaciones, por lo que el bienestar social de todos los jugadores se verá reducido.

\subsection{Estático}

La representación del juego solo con seguridad privada plantea tres supuestos; el primero consiste en que si un oportunista decide robar a un propietario pasivo se dará una transferencia de bienestar; el segundo plantea que si el propietario es activo cualquier oportunista que intente robarlo será detenido y, a su vez, castigado conforme a las penas establecidas. Por último, existe un costo de protección en el que solo incurren los propietarios activos.

Los pagos del propietario están definidos por $\{a, b, c, d\}$, los cuales cumplen con que $c>a \geq b>d$; para el oportunista los pagos están dados por $\{\alpha, \beta, \gamma, \delta\}$ que de igual manera cumplen con $\delta>\gamma \geq \alpha>\beta$.

\section{Oportunista}

\begin{tabular}{cc|cc} 
& & No Robar & Robar \\
\cline { 2 - 4 } Propietario & Activo & $a, \alpha$ & $b, \beta$ \\
& Pasivo & $c, \gamma$ & $d, \delta$
\end{tabular}


Las desigualdades para el propietario expresan que es mejor ser pasivo cuando el oportunista decide no robar, y activo cuando decide robar. Y para el oportunista es mejor no robar cuando el propietario es activo y robar cuando es pasivo. Conforme a lo anterior no se puede determinar un equilibrio de Nash en estrategias puras; sin embargo, existe uno en estrategias mixtas. ${ }^{1}$

Para determinar los equilibrios de Nash del modelo se obtienen como si fuera un juego 1 vs. 1, pero la intuición del modelo debe ser para un juego poblacional. Se asume que $P($ Activo $)=p$ y $P($ Pasivo $)=1-p$ y para el oportunista $P$ (No Robar $)=q$ y $P($ Robar $)=1-q$. De esta manera, los equilibrios de Nash en estrategias mixtas para el modelo solo con seguridad privada serán (2.2).

$$
\begin{aligned}
p^{*} & =\frac{\delta-\gamma}{\alpha+\delta-(\beta+\gamma)} \\
q^{*} & =\frac{d-b}{a+d-(b+c)}
\end{aligned}
$$

Ahora, al introducir a la policía pública los supuestos del modelo anterior se mantienen, aunque con algunas modificaciones en los pagos debido a que se introducen dos nuevos términos. El primero representa el nivel de efectividad de la policía pública $(x)$ y toma valores entre 0 y 1 , siendo 0 como totalmente inefectiva y 1 efectividad total. El segundo término es $T(x)$ y su utiliza para representar un impuesto lump-sum que paga toda la población por el servicio de seguridad pública; esta nueva variable indica que todos los jugadores estarán pagando impuestos, por lo que sus beneficios se verán reducidos en dicho valor.

\section{Oportunista}

\begin{tabular}{cc|cc} 
& & No Robar & Robar \\
\cline { 2 - 4 } Propietario & Activo & $a, \alpha$ & $b, \beta$
\end{tabular}$-T(x)$

Ahora, en el modelo anterior solo se podía detener al oportunista que robaba si el propietario era activo; sin embargo, con la introducción de $x$ existe la probabilidad de que aun cuando el propietario sea pasivo se logre la detención, lo que

1 En un juego de 1 vs. 1 el equilibrio de Nash en estrategias mixtas es la probabilidad con la que se juega una determinada estrategia, y en un juego poblacional es una proporción de la población jugando una estrategia pura. 
implicaría que $\{$ Pasivo, Robar $\}$ se convierta en un pago esperado, de tal manera que $\{d, \delta\}$ pase a ser $\{\hat{d}, \hat{\delta}\}$.

La estructura del pago esperado para el propietario es $\hat{d}=x(b+I)+(1-x) d$, donde el primer término es la probabilidad de que la policía detenga a un oportunista que decide robar $(x)$ multiplicada por el pago de haber sido activo y sufrir un robo $(b)$, más el gasto de inversión en seguridad $(I)$ que el propietario se ahorraría; el segundo término es la inefectividad de la policía $(1-x)$ por el pago de ser pasivo y sufrir un robo $(d)$. Para el oportunista será $\hat{\delta}=x(\beta)+(1-x) \delta$; el primer término expresa la efectividad de la policía $(x)$ por el pago de robary ser detenido y castigado $(\beta)$ más la inefectividad $(1-x)$ por el pago de haber robado y obtener la transferencia de bienestar $(\delta)$.

De acuerdo con el nuevo juego (2.3) las desigualdades planteadas para (2.1) no siempre se cumplen, y por tanto los equilibrios de Nash en estrategias mixtas estarán en función de $x$, por lo cual es necesario explorar estos equilibrios. Primero, si suponemos que $x=0$, los equilibrios de (2.2) se mantienen; segundo si es $x=1$, las desigualdades serán $c>a \geq \hat{d}>b$ y $\gamma \geq \alpha>\beta \geq \hat{\delta}$, lo cual implica que se obtendrá un equilibrio de Nash en estrategias puras \{Pasivo, No Robar\}. Y por último, cuando $x$ tome valores diferentes de 0 y 1 se tendrán que evaluar puntualmente los posibles equilibrios de manera iterativa, a través de los equilibrios de Nash en estrategias mixtas, los cuales ahora estarán en función de $x$ (2.4).

$$
\begin{aligned}
& p^{*}=\frac{x(\beta-\delta)+\delta-\gamma}{x(\beta-\delta)+\alpha+\delta-(\beta+\gamma)} \\
& q^{*}=\frac{x(b+I-d)+d-b}{x(b+I-d)+a+d-(b+c)}
\end{aligned}
$$

Para determinar el efecto de la seguridad pública se utilizan las ecuaciones (2.4) y se derivan respecto a $x$. El efecto de $x$ sobre los propietarios será una mayor pasividad, debido a que estos tomarán una decisión conforme a lo que creen que harán los oportunistas; por eso, estos pensarán que a mayor efectividad de la policía habrá menor número de robos y en consecuencia no es necesario ser activos ( $\mathrm{Si}$ $\alpha>\beta$ y $\delta>\beta$, Lema 1); mientras que para los oportunistas, a medida que $x$ aumenta habrá una mayor frecuencia de robos, a consecuencia del conocimiento común, es decir, ellos saben que los propietarios creerán que a mayor efectividad deberán corresponder menos robos y por ende serán más pasivos, por lo que habría más oportunidades de robo (Si $c>a$ y $b+I>d$, Lema 1). 
Es importante destacar que existe una discontinuidad en el modelo cuando $x$ toma valores considerablemente grandes y logra desincentivar totalmente a los oportunistas de robar, de tal manera que conduce al juego a un equilibrio en estrategias puras, en el cual propietarios eligen pasivo y los oportunistas no robar, de tal manera que los niveles de bienestar son discontinuos cuando $x$ es considerablemente alto. Por eso, se concluye que el Lema 1 se cumple si y solo si existe un equilibrio en estrategias mixtas.

Ahora, una vez definido el equilibrio estático del modelo (equilibrio de Nash) se determinan los beneficios del propietario y oportunista (2.5), respectivamente, y el nivel de bienestar social, el cual será la suma de los dos anteriores.

$$
\begin{aligned}
& \pi_{p r}=\frac{a \hat{d}-b c}{a+\hat{d}-(b+c)}-T \\
& \pi_{o p}=\frac{\alpha \hat{\delta}-\beta \gamma}{\alpha+\hat{\delta}-(\beta+\gamma)}-T
\end{aligned}
$$

Es claro que el pago esperado de los jugadores estará determinado por $x$ (2.5), por lo que es necesario explorar las implicaciones que tiene esta variable. Para el pago esperado de los propietarios se tendrá una reducción negativa en su nivel de bienestar ante aumentos en la efectividad de la policía, mientras que los oportunistas tendrán un ligero cambio positivo (Lema 2); esto implicaría una reducción en el nivel de bienestar social dada la suma de las variaciones. Es importante destacar que la discontinuidad del modelo también aplica para los pagos esperado, por lo cual estas conclusiones se cumplen si y solo si existe un equilibrio en estrategias mixtas, ya que como se mencionó anteriormente los niveles de bienestar son discontinuos cuando $x$ es considerablemente alto.

Hasta aquí se ha analizado de manera estática el modelo, desde sus equilibrios hasta los cambios que podría generar $x$; sin embargo, este juego debe ser visto como un proceso que está ocurriendo, en el que los jugadores aprenden a lo largo del tiempo, es decir, siguen un proceso evolutivo en sus estrategias, por lo que en la siguiente sección se determinará la dinámica del replicador. 


\subsection{Dinámica}

Este apartado se enfoca en determinar la dinámica del replicador y sus distintos estados estacionarios y estabilidad. Después se realiza un ejemplo práctico para ilustrar lo anteriormente explicado y se propone un sistema de incentivos para un estado de inseguridad.

Primero es necesario definir la dinámica a través de la cual las estrategias de los jugadores estarán evolucionando. Los aspectos teóricos para establecer en la dinámica del replicador es que esta es definida como un protocolo imitativo en el cual los jugadores reproducen la estrategia que consideran más exitosa a través de la ecuación del replicador $\dot{x}_{i}^{p}=x_{i}^{p} \hat{F}_{i}^{p}(x)$. En esta dinámica la tasa de crecimiento de cada $i \in S^{p}$ estrategia es igual al exceso de pago expresado como $\hat{F}_{i}^{p}(x)=F_{i}^{p}(x)-\bar{F}^{p}(x)$ y además se asume que $\dot{N}=0$, lo que representa que no hay nacimientos ni muertes durante el proceso evolutivo. En resumen, la dinámica del replicador permite que los jugadores cambien de estrategia hacia una más rentable mediante la comparación.

Para determinar la dinámica del juego (2.3) se obtienen las ecuaciones del replicador (2.6). Estas se representan en un diagrama de fases con $p$ en las abscisas y 1-q en las ordenadas, y se explica cómo cambian las frecuencias de elección entre activo y robar, es decir, la correspondencia entre los niveles de propietarios activos y oportunistas robando.

$$
\begin{aligned}
& p^{*}=p(1-p)(b-\hat{d}-(b+c-(a+\hat{d})) q) \\
& q^{*}=q(1-q)(\gamma-\hat{\delta}-(\gamma+\beta-(\alpha+\hat{\delta})) p)
\end{aligned}
$$

Ejemplo.

\section{Oportunista}

\begin{tabular}{cc|cc} 
& & No Robar & Robar \\
\cline { 2 - 4 } Propietario & Activo & 3,2 & 2,1 \\
& Pasivo & 4,3 & 1,4
\end{tabular}

Los pagos expresados en (2.7) son definidos conforme a las desigualdades de (2.1) y con un Eq. de Nash $=\left\{p^{*=1 / 2}, q^{*=1 / 2}\right\}$. Los valores de $p$ y $q$ representan 
la frecuencia de la población jugando activo y no robar, respectivamente. Si decidiéramos graficar la correspondencia entre poblaciones con activos en el eje de las abscisas y robar en las ordenadas, con valores de entre 0 y 1 , nos situaríamos justamente en el centro, con una mitad de propietarios activos y una de oportunistas robando.

Por lo anterior, se pueden plantear cuatro condiciones para la población según sus estrategias. Paz: $p^{*}<1 / 2, q^{*}>1 / 2$, Inseguridad: $p^{*}<1 / 2, q^{*}<1 / 2$, Seguridad: $p^{*}>1 / 2, q^{*}>1 / 2$ y Conflicto: $p^{*}>1 / 2, q^{*}<1 / 2$, donde cada una representa la correspondencia entre jugadores. La primera es un entorno en el cual no hay robos y por ende no es necesario protegerse, la segunda muestra un estado con una alta tasa de delincuencia y baja protección, la tercera es el caso totalmente contrario al anterior, baja delincuencia y alta protección, y para finalizar se tiene un entorno en el que hay un constante enfrentamiento dado el alto número de robos y propietarios activos.

Conforme a lo anterior, este trabajo se enfoca en estudiar una población en condiciones de inseguridad a través de una serie de modificaciones en los pagos. El objetivo es ver qué ocurre con los beneficios, la dinámica del replicador y las intervenciones gubernamentales.

\section{Condición de inseguridad}

El modelo presentado tiene, de manera implícita, una serie de términos detrás que explica la magnitud de los pagos; sin embargo, con la finalidad de que se pueda comprender mejor y estudiar las implicaciones de políticas públicas se redefinieron e incluyeron algunos más. Se introducen dos términos $W_{1}$ y $W_{2}$, los cuales representan el nivel de bienestar que el propietario pierde y el oportunista gana, respectivamente, cuando ocurre un robo. Para dar una explicación más intuitiva y sea más simple modificar los incentivos se replantearon algunos pagos incluyendo algunas variables. Por ejemplo, $d$ era expresada como $-D$ y fue modificada a $V-D-W_{1}$ para representar que del nivel de bienestar del propietario $(V)$ se le debe restar el daño que este sufre $(D)$ y la pérdida de bienestar $\left(W_{1}\right)$.

Se realizó una serie de modificaciones a los pagos de (2.7), de tal manera que permitan representar un entorno en las condiciones deseadas. Primero se detalló la situación del sistema de seguridad, y conforme a Becker (1968) se consideran bajas penalidades $(F)$, por lo que habría incentivos de robar por parte de los oportunistas. En términos de la sociedad se incluye la existencia de desigualdad 


\section{Oportunista}

\begin{tabular}{cc|cc} 
& & No Robar & Robar \\
\cline { 2 - 4 } Propietario & Activo & $V-I, U-E$ & $V-I-D, U-F$ \\
& Pasivo & $V, U$ & $V-D-W_{1}, U+W_{2}$
\end{tabular}

$(V, U)$, cuyo efecto en el crimen tendería a aumentar la frecuencia de los robos debido a la existencia de mayores y mejores oportunidades de robo (Vila y Cohen, 1993). Segundo, se proponen crear propietarios poco alertas $(E)$ y temerosos $(D)$, con la finalidad de que no tiendan a ser activos, sino cautivos en la inseguridad. Un detalle adicional que ya presentaba el modelo anterior es una transferencia desigual $W_{1}>W_{2}$, lo que implicaría que los niveles de bienestar perdidos de los propietarios sean mayores a los obtenidos por el oportunista.

Se presenta un nuevo sistema de incentivos conforme a las modificaciones señaladas. Para la situación de la sociedad se redujeron las penas por robo $(F)$ y se acrecentó la desigualdad con una reducción en el salario legal de los oportunistas $(U)$. Sobre las características de los propietarios se eliminó el costo de sospecha $(E)$ y aumentó el daño hacia los propietarios por sufrir un robo $(D)$. Las variables de transferencia de bienestar $\left(W_{i}\right)$ aumentaron, pero respetando la proporción existente. De acuerdo con estas modificaciones se concluye el juego (3.2) para el cual se cumplen las desigualdades mencionadas.

\section{Oportunista}

\begin{tabular}{cc|cc} 
& & No Robar & Robar \\
\cline { 2 - 4 } Propietario & Activo & 8,7 & 5,5 \\
& Pasivo & 10,7 & 4,8
\end{tabular}

Para (3.2) se obtuvo un Eq. de Nash $=\left\{p^{*}=1 / 3, q^{*}=1 / 3\right\}$, que expresa un mayor número de propietarios pasivos y oportunistas robando, lo cual clasifica este equilibrio dentro de la condición de inseguridad. Para fines prácticos se propone una gráfica para el plano de condiciones que muestre la correspondencia entre activos y robando con $(p, 1-q)$ en los ejes. Las cuatro condiciones de la población se ven reflejadas en la Gráfica 1 y se observa que el equilibrio de Nash se sitúa en la parte de inseguridad. 
Gráfica 1. Plano de condiciones

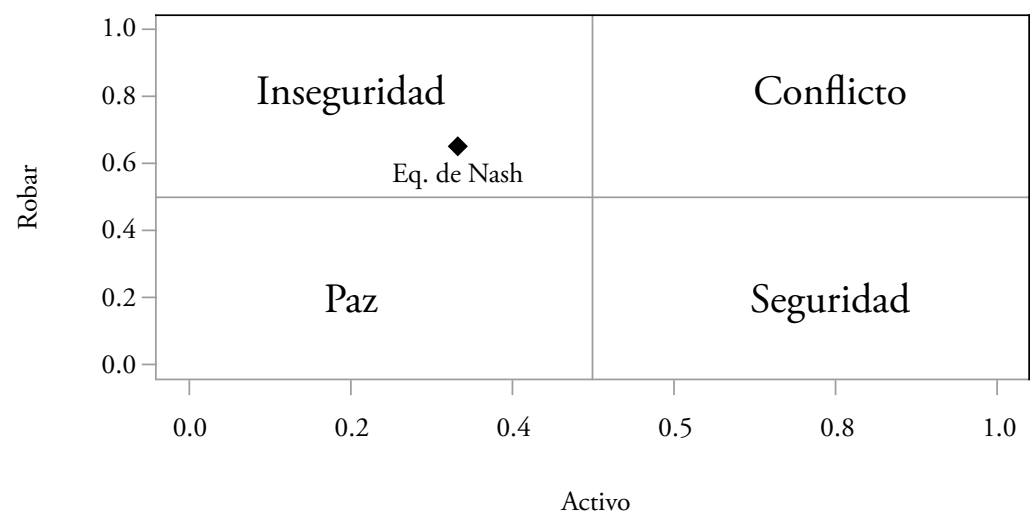

\subsection{Dinámica}

En esta sección se estudia el plantea miento del juego con seguridad pública y privada mediante la dinámica del replicador para diferentes niveles de, sus equilibrios de Nash y estados estacionarios.

\section{Oportunista}

\begin{tabular}{cc|cc}
\multirow{2}{*}{ Propietario } & & No Robar & Robar \\
\cline { 2 - 4 } & Activo & 8,7 & 5,5 \\
& Pasivo & 10,7 & $2 x+4,8-3 x$
\end{tabular}

Se determinan las ecuaciones del replicador para $x=[0,0.10,0.25,0.40]$; el primer valor es para el juego sin gobierno (3.2) y los otros tres son niveles similares a los utilizados en el trabajo original (Cressman et al., 1998). En la Gráfica 2 se muestra la dinámica del replicador para la condición inicial $\left(p_{0}, q_{0}\right)=(0.5,0.5)$ y para $x=[0,0.40]$, no se grafican los valores 0.10 y 0.25 porque no aportan un comportamiento diferente a $x=0$, solamente un equilibrio de Nash que se mueve hacia la parte superior izquierda. Se muestra un semicírculo que gira alrededor del equilibrio de Nash y que mayormente oscila dentro de la condición de inseguridad; esta trayectoria pertenece a $x=0$. Y para $x=0.40$ existe una trayectoria de convergencia hacia $\{p=0, q=1\}$, es decir, pasivo y no robar. 
Gráfica 2. Dinámica del replicador

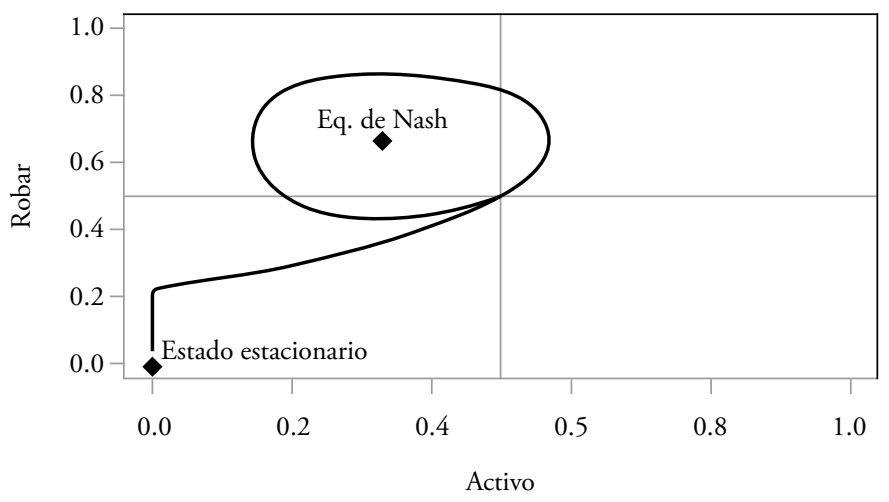

Para los sistemas de $x=[0,0.10,0.25]$ se obtuvieron cinco estados estacionarios, cuatro situados en los vértices y uno en el equilibro de Nash del juego; los estados de los vértices corresponden a puntos inestables, mientras que el equilibrio de Nash es un círculo concéntrico alrededor del cual gira la dinámica del replicador (Friedman, 1998), como se muestra en la Gráfica 2.

En términos aplicados se puede decir que la población estará girando alrededor de los equilibrios de Nash correspondientes a cada valor de $x$. Por eso, como tal, la población no converge en ningún valor, solamente estará en un "círculo vicioso", y cuando los propietarios perciban una alta frecuencia de oportunistas robando optarán por protegerse; a medida que haya mayor protección se reducirán los robos, lo cual, a su vez, hará que los propietarios sientan confianza por la baja en los robos y comenzarán a dejar de ser activos, hasta el punto en que los oportunistas vean esto como una oportunidad de robo y comiencen a actuar, y así sucesivamente en un círculo, que es más grande para valores más grandes de, siempre y cuando estos se encuentren en el intervalo [0.1/3].

Las implicaciones que tiene $x$ sobre los equilibrios de Nash es diferente para cada población, y depende de qué tan grande sea $x$. Si $x<1 / 3$ tendrá un efecto de pasividad en los propietarios, mientras que en los oportunistas incentivará la frecuencia de robo, tal como se observa en la Gráfica 2, pareciera que los equilibrios de Nash convergerán en un estado en el cual todos opten por robar y no haya propietarios activos; sin embargo, esto no ocurre, dado que el equilibrio de Nash es discontinuo. 
Esta discontinuidad se refiere a que el equilibrio de Nash está en función de la policía pública y cuando $x>1 / 3$, robar se vuelve una estrategia estrictamente dominada, y por ende todos los oportunistas pierden incentivos para robar y la población converge en $\{p=0, q=1\}$, como se observa en la Gráfica 2. Esto se explica a través de los incentivos; cuando la seguridad pública es muy alta, y por tanto los policías son muy efectivos y logran detener a un tercio de los oportunistas que deciden robar, la respuesta será una reducción inmediata en el pago esperado de estos, ya que la probabilidad de que sean detenidos es muy alta, y correr el riesgo de ser castigados no es una estrategia viable; en consecuencia, la tasa de robos comenzará a bajar y en respuesta a esto los propietarios dejarán de ser activos debido a que no hay nada de qué protegerse, por lo cual en el equilibrio de Nash no existirá inseguridad. Es decir, para los equilibrios de Nash en los que $x<1 / 3$ pareciera que se tiende a $\{p=0, q=0\}$, pero cuando $x$ crece lo suficiente para desincentivar el robo, el equilibrio de Nash se vuelve un estado estacionario estable.

En resumen, la población comenzará situada en un estado neutral, con una diferencia de bienestar desigual, un sistema penal relajado, altos costos por sufrir un robo, propietarios temerosos y una transferencia de bienestar desigual, lo que propiciará un alto nivel de pasividad $\left(p^{*}<0.5\right)$, que aprovecharán los oportunistas para mantener una alta frecuencia de robos $\left(q^{*}<0.5\right)$, y dado que los propietarios serán cautivos y la efectividad aumentará en niveles ineficientes, parecerá que se tiende a un estado de inseguridad extremo $(p=0, q=0)$; sin embargo, cuando $x$ sea mayor a $1 / 3$ los oportunistas perderán todos los incentivos para robar, dado que la probabilidad de ser detenidos será muy alta para su análisis costo-beneficio y, por ende, preferirán conservar su sueldo legal y evitar los castigos, lo cual los llevará hacia una estado estacionario que maximizará el bienestar social.

\section{Intervención gubernamental y bienestar social}

En este modelo la única función del gobierno es crear intervenciones que aseguren el máximo bienestar social de la población a través de mecanismos como aumentos en la efectividad y las penalidades. Para estudiar los medios de contención del robo se propone un incremento en las penalidades acompańado de un aumento en $x$, y se determina si estas políticas complementarias permiten alcanzar un estado estacionario en valores menores de seguridad pública. 
Para determinar si las penalidades tienen un impacto en el equilibrio de Nash se recurre a la literatura (Becker, 1968; Ehrlich, 1996; Eide, 1997; Grautoff et al., 2018)en la que se demuestra que la frecuencia de robo es inelástica ante cambios en las penalidades, argumento que este modelo también valida (Lema 3). Esto es sencillo de demostrar debido a que $q^{*}$ no está en función de $\beta$, pago que incluye las penalidades, por lo que los robos mantendrían su mismo nivel; sin embargo, en los propietarios hay un mecanismo diferente, pues al saber que hay mayores penalidades esperarán a que los oportunistas reduzcan la frecuencia de robos y por ende sea menos necesario protegerse, lo que llevaría a una reducción de $p^{*}$ (Lema 3). En resumen, cambios en las penalidades no afectan la frecuencia de los robos, sino que generan pasividad en los propietarios debido al conocimiento común, de tal manera que solamente el equilibrio de Nash se desplazará a la izquierda.

Ahora bien, para determinar si el aumento en las penalidades tiene un impacto en el bienestar social se hace uso del Lema 3. En este se demuestra que el bienestar social no tiene variaciones por cambios en las penalidades; esto se debe a que el pago esperado del propietario $\pi_{p r}$ no está en función de $\beta$ y que el pago esperado del oportunista es inelástico a $\beta$. Por tanto, un aumento en las penalidades no modifica el bienestar social del modelo; es decir, si el gobierno busca maximizar el bienestar social a través de modificaciones en el sistema judicial que incremente las penas a los que cometan actividades delictivas no tendrán impacto alguno, por lo que su único mecanismo viable de intervención será el aumento en la seguridad pública.

Para demostrar la importancia de $x$ en el bienestar social se graficó el pago esperado para el propietario (puntos y círculos) y el del oportunista (línea) para todos los valores que puede tomar la seguridad pública. En la Gráfica 3 se obtiene una línea recta con pendiente negativa para $x<1 / 3$ para el pago esperado del propietario y después de este valor se tiene un salto (discontinuidad, como se mencionó anteriormente), debido a que cuando $x$ es suficientemente grande se alcanza el equilibrio de Nash en el que todos los propietarios son pasivos y los oportunistas deciden no robar; este se define como el máximo de bienestar social. Para el oportunista existe una particularidad que, como ya se comentó, los niveles de $x$ tienen un ligero impacto en su pago esperado (Lema 2), pero al aumentar en términos tan pequeños reduce este impacto a 0 , por lo que este se mantiene constante e igual a 7 para todos los valores de $x$.

Se concluye que para una población en condiciones de inseguridad son necesarios cambios en la efectividad lo suficientemente grandes para disuadir el robo, 


\section{Gráfica 3. Pago esperado}

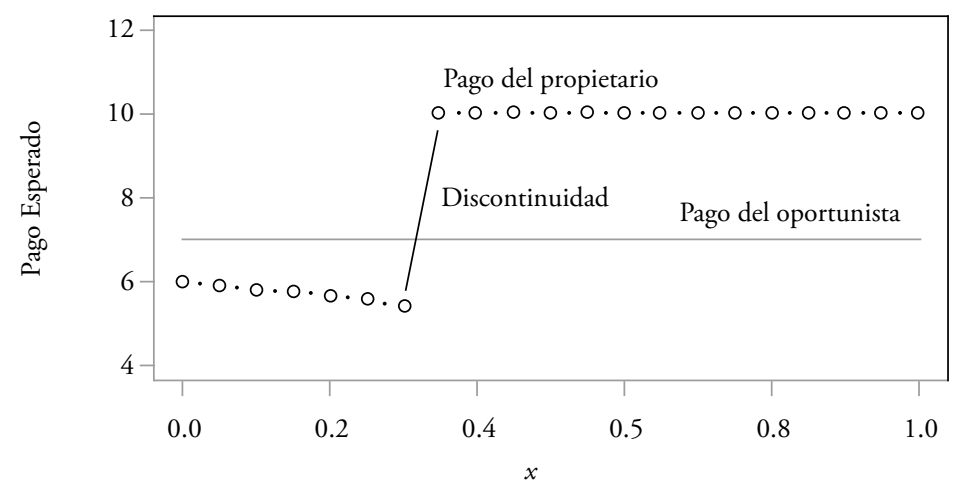

independientemente de los aumentos en las penalidades. Y se evidenció que si estos aumentos en $x$ no logran disuadir el robo y solamente son un ligero incremento inefectivo, el bienestar social estará disminuyendo debido a que los propietarios tendrán descensos en su pago esperado a consecuencia del conocimiento común.

\section{Conclusiones}

El objetivo de este ejercicio teórico es determinar los efectos de las distintas variables que se tienen alrededor de la actuación de la seguridad pública, en un contexto de la teoría de los juegos evolutivos. Como se menciona, este juego toma en cuenta, por un lado, los costos de implementación de un sistema de penas y un nivel de vigilancia óptimos, mientras que en el otro aspecto se considera la capacidad de los criminales de decidir el mejor momento y los ciudadanos o propietarios que buscarán proteger sus intereses. Un escenario con un alto nivel de inseguridad llevaría a una disminución en el nivel de bienestar de los propietarios que tendrían que asumir mayores costos de protección privada, además de los costos que ya debe asumir como sociedad por el pago de la seguridad pública. Este es el resultado básico que se extrae del modelo estático.

Ante un modelo dinámico, nos encontramos ante una situación en la cual ambos jugadores adaptan sus estrategias dependiendo de los resultados que va arrojando el juego. Este tipo de análisis permite determinar qué tan efectivas 
o no son las acciones en búsqueda de un estado de seguridad, ya sea por medio de mayores inversiones en seguridad pública, o bien en intervenciones por parte del gobierno sobre los criminales. En una situación ideal, el mejor resultado para la sociedad se obtiene cuando los ciudadanos no deben protegerse y los criminales no tienen incentivos netos para delinquir (beneficio de la actividad criminal menos pérdidas en caso de ser detenido). La efectividad en la actuación policial suele estar limitada, en el mundo real, por la cifra negra (tasa de no denuncias) a la que se enfrentan. Si el monto del robo es menor al costo de oportunidad de hacer la denuncia, la ciudadanía prefiere no hacerlo formalmente, con lo cual el costo para el criminal se limita a una detención momentánea. Otra limitación es el número de fuerza policíaca necesaria para cubrir las necesidades óptimas de seguridad, donde el costo de protegerse sea nulo o bajo respeto al riesgo de sufrir un hecho delictivo. Asimismo, las penas a las que se enfrenta el criminal, una vez que se le detiene y presenta la denuncia, deben implicar beneficios negativos para el delincuente ante la ganancia que obtiene por efectuar su actividad contra la ciudadanía.

De esta forma llegamos a una pregunta: ¿que es mejor, mayor seguridad pública o penas mayores? Para el bienestar social, es decir de la ciudadanía, una mayor seguridad pública implicaría la posibilidad de un menor gasto en protección privada: sin embargo, esto solo sería una forma donde se limitarían los incentivos de los delincuentes, con lo cual un incremento en las penas actuaría como complemento para buscar reducir aún más esos incentivos a la actividad criminal, siempre teniendo en mente el bienestar social.

\section{Referencias}

Becker, G. S. (1968). Crime and Punishment: An Economic Approach. Journal of Political Economy, 49.

Cohen, L. E. y Machalek, R. (1988). A General Theory of Expropriative Crime: An Evolutionary Ecological Approach. American Journal of Sociology, 94(3), 465-501.

Cressman, R., Morrison, W. G. y Wen, J.-F. (1998). On the Evolutionary Dynamics of Crime. The Canadian Journal of Economics / Revue Canadienne d'Economique, 31(5), 1101-1117. https://doi.org/10.2307/136461

Ehrlich, I. (1996). Crime, Punishment, and the Market for Offenses. The Journal of Economic Perspectives, 10(1), 43-67. 
Eide, E. (1997). Economics of Criminal Behavior. Encyclopedia of Law y Economics, 45.

Friedman, D. (1998). Evolutionary economics goes mainstream: a review of the theory of learning in games. Journal of Evolutionary Economics, 8(4), 423-432.

Grautoff, M., Chavarro, F. y Arce, A. F. (2018). La Teoría Racional del Crimen. Aplicaciones de Gary Becker en Bogotá, D.C. Criterio Libre, 9(14), 91-124. https://doi.org/10.18041/1900-0642/criteriolibre.2011v9n14.1233

Machalek, R. y Cohen, L. E. (1991). The nature of crime: Is cheating necessary for cooperation? Human Nature, 2(3), 215-233. https://doi. org/10.1007/BF02692187

Mealey, L. (1997). An Evolutionary, But Not Stable Strategy for Crime Control. Politics and the Life Sciences, 16(1), 38-39. https://doi.org/10.1017/ S0730938400020220

Nash, J. (1950). Non Cooperative Games. Princeton.

Savage, J. y Vila, B. (2003). Human ecology, crime, and crime control: Linking individual behavior and aggregate crime. Biodemography and Social Biology, 50(1-2), 77-101. https://doi.org/10.1080/19485565.2003.9 989066

Vila, B. (1994). A General Paradigm For Understanding Criminal Behavior: Extending Evolutionary Ecological Theory. Criminology, 32(3), 311360. https://doi.org/10.1111/j.1745-9125.1994.tb01157.x

Vila, B. (1995). Expropriative Crime and Crime Policy: An Evolutionary Ecological Analysis, 24.

Vila, B. (1997). Human Nature and Crime Control: Improving the Feasibility of Nurturant Strategies. Politics and the Life Sciences, 16(1), 3-21. https://doi.org/10.1017/S0730938400020153

Vila, B. J. y Cohen, L. E. (1993). Crime as Strategy: Testing an Evolutionary Ecological Theory of Expropriative Crime. American Journal of Sociology, 98(4), 873-912. https://doi.org/10.1086/230092

Weissing, F. y Ostrom, E. (1991). Crime and Punishment: Further Reflections on the Counterintuitive Results of Mixed Equilibria Games. Journal of Theoretical Politics, 3(3), 343-350. https://doi. org/10.1177/0951692891003003006 


\section{Apéndice}

Lema 1. Si aumenta la efectividad de la policía los propietarios serán más pasivos y los oportunistas aumentarán su frecuencia de robo.

Propietario. El aumento de la vigilancia de la policía genera pasividad en los propietarios por medio de dos efectos: el directo de $x$ sobre $\hat{\delta}$ y el indirecto por medio del conocimiento común definido por $\hat{\delta}$ y $p^{*}$.

$$
\frac{d p^{*}}{d x}=\frac{d p^{*}}{d \hat{\delta}}\left(\frac{d \hat{\delta}}{d x}\right)<0 \text { dado que } \frac{d p^{*}}{d \hat{\delta}}>0, \frac{d \hat{\delta}}{d x}<0
$$

El efecto directo es negativo porque un aumento de $x$ hace más probable que el oportunista sea detenido y, por consecuencia, el pago esperado sea menor, dado que $\delta>\beta$.

$$
\frac{d \hat{\delta}}{d x}=\beta-\delta<0
$$

Y el efecto del conocimiento común está dado porque el propietario supone que ante un mayor patrullaje el oportunista reducirá la frecuencia de robo y, por tanto, se reducirá su protección, dado $\alpha>\beta$.

$$
\frac{d p^{*}}{d \hat{\delta}}=\frac{\alpha-\beta}{(\alpha+\hat{\delta}-(\beta+\gamma))^{2}}>0
$$

Oportunistas. El aumento de la vigilancia de la policía genera una mayor frecuencia de robos por medio de dos efectos: el directo de $x$ sobre $\hat{d}$ y el indirecto por medio del conocimiento común definido por $\hat{d}$ y $q^{*}$.

$$
\frac{d q^{*}}{d x}=\frac{d q^{*}}{d \hat{d}}\left(\frac{d \hat{d}}{d x}\right)<0 \text { dado que } \frac{d q^{*}}{d \hat{d}}<0, \frac{d \hat{d}}{d x}>0
$$


El efecto directo es positivo, debido a que una mayor vigilancia el pago esperado de un oportunista pasivo aumentaría, dado $b+I<d$.

$$
\frac{d \hat{d}}{d x}=b+I-d>0
$$

Mientras que el efecto del conocimiento común esta explicado porque el oportunista supone que a mayor vigilancia se generará pasividad y, en consecuencia, mayores oportunidades, dado $c<a$.

$$
\frac{d q^{*}}{d \hat{d}}=\frac{a-c}{(a+\hat{d}-(b+c))^{2}}<0
$$

Lema 2. Ante aumentos en $x$ el bienestar de los propietarios se verá reducido y el de los oportunistas aumentará ligeramente.

El aumento en la vigilancia impactará de manera negativa el bienestar en los propietarios y de forma positiva el de los oportunistas por medio de cambios en el equilibrio de Nash.

$$
\frac{d \pi_{p r}}{d p^{*}}>0, \frac{d \pi_{o p}}{d p^{*}}<0
$$

Dado que

$$
\begin{aligned}
& \pi_{p r}=p^{*}\left(q^{*}\right)(a)+p^{*}\left(1-q^{*}\right)(b)+\left(1-p^{*}\right) q^{*}(c)+\left(1-p^{*}\right)\left(1-q^{*}\right)(\hat{d}) \\
& \pi_{o p}=p^{*}\left(q^{*}\right)(\alpha)+p^{*}\left(1-q^{*}\right)(\beta)+\left(1-p^{*}\right) q^{*}(\gamma)+\left(1-p^{*}\right)\left(1-q^{*}\right)(\hat{\delta})
\end{aligned}
$$

La primera derivada será positiva dado que $c<a, b<d$ y $q^{*}<\left(1-q^{*}\right)$, y la segunda será negativa dado que $\alpha>\beta, \gamma<\hat{\delta}$ y $p^{*}<\left(1-p^{*}\right)$.

$$
\begin{aligned}
& \frac{d \pi_{o p}}{d p^{*}}=q^{*}(a-c)+\left(1-q^{*}\right)(b-d)>0 \\
& \frac{d \pi_{o p}}{d q^{*}}=p^{*}(\alpha-\beta)+\left(1-p^{*}\right)(\gamma-\hat{\delta})<0
\end{aligned}
$$


En resumen, si $x$ aumenta disminuye $p^{*}(0.1)$ y $q^{*}(0.4)$, y por lo tanto reduce $\pi_{p r}$ y aumenta $\pi_{o p}$.

Lema 3. Un aumento en las penalidades solo modifica $p^{*}$ de manera positiva, ya que $q^{*}$ es inelástica y $\boldsymbol{\pi}_{o p}$ será cercano a 0 .

Dada la especificación de $p^{*}$ un aumento en las penalidades reducirá $\beta$ y el impacto de esta sobre el equilibrio de Nash es positivo, dado $\hat{\delta}>\gamma$.

$$
\frac{d p^{*}}{d \beta}=\frac{\hat{\delta}-\gamma}{(\alpha+\hat{\delta}-(\beta+\gamma))^{2}}>0
$$

Una reducción de $\beta$ disminuirá o mantendrá constante $\pi_{o p}$ dependiendo de si $\gamma \geq \alpha$.

$$
\frac{d \pi_{o p}}{d \beta}=\frac{(\gamma-\alpha)(\gamma-\hat{\delta})}{(\alpha+\hat{\delta}-(\beta+\gamma))^{2}} \leq 0
$$

